TAIWANESE JOURNAL OF MATHEMATICS

Vol. 17, No. 6, pp. 1855-1867, December 2013

DOI: $10.11650 /$ tjm.17.2013.3139

This paper is available online at http://journal.taiwanmathsoc.org.tw

\title{
HOMOCLINIC ORBITS OF NONPERIODIC SUPERQUADRATIC HAMILTONIAN SYSTEM
}

\author{
Jian Zhang, Xianhua Tang* and Wen Zhang
}

\begin{abstract}
In this paper, we study the following first-order nonperiodic Hamiltonian system

$$
\dot{z}=\mathcal{J} H_{z}(t, z),
$$

where $H \in C^{1}\left(\mathbb{R} \times \mathbb{R}^{2 N}, \mathbb{R}\right)$ is the form $H(t, z)=\frac{1}{2} L(t) z \cdot z+R(t, z)$. Under weak superquadratic condition on the nonlinearitiy. By applying the generalized Nehari manifold method developed recently by Szulkin and Weth, we prove the existence of homoclinic orbits, which are ground state solutions for above system.
\end{abstract}

\section{Introduction AND Main Results}

We study the following first-order Hamiltonian system

$$
\dot{z}=\mathcal{J} H_{z}(t, z),
$$

where $z=(p, q) \in \mathbb{R}^{N} \times \mathbb{R}^{N}=\mathbb{R}^{2 N}, \mathcal{J}=\left(\begin{array}{cc}0 & I_{N} \\ -I_{N} & 0\end{array}\right)$, and $H \in C^{1}\left(\mathbb{R} \times \mathbb{R}^{2 N}, \mathbb{R}\right)$ is the form

$$
H(t, z)=\frac{1}{2} L(t) z \cdot z+R(t, z)
$$

with $L(t) \in C\left(\mathbb{R}, \mathbb{R}^{4 N^{2}}\right)$ being a $2 N \times 2 N$ symmetric matrix valued function, and $R \in C^{1}\left(\mathbb{R} \times \mathbb{R}^{2 N}, \mathbb{R}\right)$ is superquadratic at infinity. In this paper, we are concerned with the existence of homoclinic orbits, which are ground state solutions of system $(H S)$, i.e., solutions corresponding to the least energy of the energy functional of system

Received April 2, 2013, accepted April 24, 2013.

Communicated by Eiji Yanagida.

2010 Mathematics Subject Classification: 37K05.

Key words and phrases: Homoclinic orbits, First-order Hamiltonian system, Ground state solutions, Generalized Nehari manifold.

This work is partially supported by the NNSF (No. 11171351) and the SRFDP (No. 20120162110021)

of China and Hunan Provincial Innovation Foundation for Postgraduate.

*Corresponding author. 
$(H S)$. Here by a homoclinic orbit of system $(H S)$ we mean a solution of the equation satisfying $z(t) \not \equiv 0$ and $z(t) \rightarrow 0$ as $|t| \rightarrow \infty$.

Establishing the existence of homoclinic orbits for system like $(H S)$ is one of the most important problems in the theory of Hamiltonian systems. In very recent years, many authors devoted to the existence of homoclinic orbits for Hamiltonian systems via critical point theory. For example, see [2, 4, 6, 14, 16, 24] for the second order systems, and $[1,3,5,7-11,13,15,17-21]$ for the first order systems. Coti-Zelati, Ekeland and Seré first considered the system $(H S)$ in [1]. Under the Ambrosetti-Rabinowitz growth condition, they proved the existence and multiplicity of homoclinic orbits for strictly convex Hamiltonian system. The existence of infinitely many homoclinic orbits was established in Seré [3], which generalized the result in [1]. Subsequently, Hofer and Wysocki [13] removed the convexity assumption and obtained the existence of homoclinic orbits. Using a subharmonic approach, Tanaka [19] also removed the convexity assumption, and proved that the system $(H S)$ has at least one homoclinic orbit. Later, suppose that $R(t, z)$ and $L(t)$ depend periodically on $t$, the existence of homoclinic orbit for system $(H S)$ was considered in [5, 7, 11, 18, 15] and [20].

Without assumption of periodicity the problem is quite different in nature, and the main difficulty of such type problem is the lack of compactness of the Sobolev embedding theorem. In [9], Ding and Li first obtained one homoclinic orbits for the nonperiodic system $(H S)$ with the compactness conditions. Recently, Ding and Jeanjean [8] imposed a control on the size of $R(t, z)$ with respect to the behavior of $L(t)$ at infinity in $t$ to recover sufficient compactness, and obtained certain existence and multiplicity results for system $(H S)$ when $R(t, z)$ is asymptotically quadratic in $z$ at infinity. Soon after, Ding and Lee [10] studied superquadratic case and obtained the existence of homoclinic orbit for system $(H S)$ by considering certain auxiliary problem related to the "limit equation". For other results about nonperiodic case, we refer readers to [21] and references therein.

To continue the discussion, we define some notations. For any real function $U(x)$ will be regarded as a symmetric matrix $U(x) I_{2 N \times 2 N}$ and $\mathcal{J}_{0}:=\left(\begin{array}{cc}0 & I_{N} \\ I_{N} & 0\end{array}\right)$, for two given matrix valued functions $M_{1}(t)$ and $M_{2}(t)$, we say that $M_{1}(t) \leq M_{2}(t)$ if and only if

$$
\max _{\xi \in \mathbb{R}^{2 N},|\xi|=1}\left(M_{1}(t)-M_{2}(t)\right) \xi \cdot \xi \leq 0
$$

and $M_{1}(t)>M_{2}(t)$ if and only if $M_{1}(t) \leq M_{2}(t)$ does not hold. Here we will mention the recent work of Chen and Ma [15]. Based on the main ideas of [25] and [29], they obtained the existence of ground state solutions by using variant generalized weak linking theorem for a strongly indefinite problem developed by Schechter and Zou [30] for the periodic case. Motivated by the above fact, in this paper our aim is to consider the nonperiodic case, i.e, $L(t)$ and $R(t, z)$ are nonperiodic with respect 
to $t$, and also establish the existence of ground state solutions by generalized Nehari manifold method developed recently by Szulkin and Weth [25](see also [27]). To our knowledge, there is no work focused on this case. Compared to the periodic case, the nonperiodic case becomes more complex, there are some difficulties to overcome. Firstly, the main difficulty of such type problem is the lack of compactness of the Sobolev embedding theorem. In order to overcome this difficulty, we assume that $L(t)$ satisfies

$\left(L_{0}\right) L(t) \in C\left(\mathbb{R}, \mathbb{R}^{2 N \times 2 N}\right)$, there exists $r_{0}>0$ such that, for any $h>0$,

$$
\text { meas }\left(\left\{t \in \mathbb{R}:\left|t-t_{1}\right| \leq r_{0}, \quad \mathcal{J}_{0} L(t)<h\right\}\right) \rightarrow 0 \text {, as }\left|t_{1}\right| \rightarrow \infty,
$$

where meas $(\cdot)$ denotes the Lebesgue measure. Secondly, since the energy functional associated to system $(H S)$ is strongly indefinite under the assumption $\left(L_{0}\right)$. Therefore, the usual Nehari manifold method cannot be applied directly (see [27]). To overcome the strongly indefiniteness of the energy functional, we will use the generalized Nehari manifold method.

More Precisely, we make the following assumptions:

$\left(R_{1}\right) R \in C^{1}\left(\mathbb{R} \times \mathbb{R}^{2 N},[0, \infty)\right)$ and $R(t, z)>0$ for all $z \neq 0$;

$\left(R_{2}\right)\left|R_{z}(t, z)\right| \leq C\left(1+|z|^{p-1}\right)$ for some $C>0, p>2$ and $R_{z}(t, z)=o(|z|)$ as $|z| \rightarrow 0$ uniformly in $t$;

$\left(R_{3}\right) \frac{R(t, z)}{|z|^{2}} \rightarrow \infty$ as $|z| \rightarrow \infty$ uniformly in $t$, and $\hat{R}(t, z):=\frac{1}{2} R_{z}(t, z) \cdot z-R(t, z)>0$ for all $z \neq 0$;

$\left(R_{4}\right)\left(R_{z}(t, z) \cdot w\right)(z \cdot w) \geq 0$ uniformly in $t$ for all $z, w \in \mathbb{R}^{2 N}$, where $z \cdot w$ denotes the usual Euclidean scalar product;

( $\left.R_{5}\right) R(t, z)=R(t, w)$ and $R_{z}(t, z) \cdot w \leq R_{z}(t, z) \cdot z$ uniformly in $t$ if $|z|=|w|$, if in addition $z \neq w$, then $R_{z}(t, z) \cdot w<R_{z}(t, z) \cdot z$;

$\left(R_{6}\right) R_{z}(t, z) \cdot w \neq R_{z}(t, w) \cdot z$ uniformly in $t$ if $|z| \neq|w|$ and $z \cdot w \neq 0$. ple:

For the nonlinearity $R(t, z)$, there are some functions satisfy $\left(R_{1}\right)-\left(R_{6}\right)$, for exam-

Ex. 1. Let $R(t, z)=f(t)|z|^{p}$, where $p>2$ and $f(t)>0$.

Ex. 2. Let $R(t, z)=g(t)\left(|z|^{p}+(p-2)|z|^{p-\varepsilon} \sin ^{2}\left(\frac{|z|^{\varepsilon}}{\varepsilon}\right)\right)$, where $g(t)>0,0<\varepsilon<$ $p-2$. Note that

$$
R_{z}(t, z)=g(t) z\left[(p-2)(p-\varepsilon)|z|^{p-\varepsilon-2} \sin ^{2}\left(\frac{|z|^{\varepsilon}}{\varepsilon}\right)+\left(p+(p-2) \sin \left(\frac{2|z|^{\varepsilon}}{\varepsilon}\right)\right)|z|^{p-2}\right] .
$$

It is easy to show that $R(t, z)$ does not satisfy the Ambrosetti-Rabinowitz superquadratic condition.

Our main result is the following: 
Theorem 1.1. Let $\left(L_{0}\right),\left(R_{1}\right)-\left(R_{6}\right)$ be satisfied, the system $(H S)$ has at least one homoclinic orbit, which is a ground state solution.

It is well known that without Ambrosetti-Rabinowitz condition, such problems become quite difficult and complex. There are some papers considered the existence of ground states for other problems, e.g., the Schrodinger equation, the elliptic system and the second-order Hamiltonian system. Szulkin and Weth [25] first obtained a ground state solutions via generalized Nehari manifold under the stronger Nehari condition. Later, Liu [23] generalized the result in [25] by relaxing the Nehari condition. Also, note that some authors have studied several different problems by a variant generalized weak linking theorem and monotonicity trick developed by Schechter and Zou [30]. Among these problems are the Schrödinger equation with spectrum zero in Yang et al. [28], the Schrodinger equation without spectrum zero in Yang [29], the elliptic system in Zhao et al. [22] and the second-order Hamiltonian system in Chen and Ma [24].

The remainder of this paper is organized as follows. In section 2 , the variational setting and the method of the generalized Nehari manifold are briefly presented. The existence of a ground state solution is proved in section 3 .

\section{Variational Setting and Generalized Nehari Manifold Method}

Below by $|\cdot|_{q}$ we denote the usual $L^{q}$ - norm, $(\cdot, \cdot)_{2}$ denote the usual $L^{2}$ inner product, $c_{i}, C, C_{i}$ stand for different positive constants. For convenience, let Hamiltonian operator

$$
A=-\left(\mathcal{J} \frac{d}{d t}+L(t)\right),
$$

and let $\sigma(A), \sigma_{d}(A)$ be the spectrum of $A$, the discrete spectrum of $A$, respectively. Observe that, since we have assumed $\left(L_{0}\right)$ about $L(t), A$ is a selfdajoint operater on $L^{2}:=L^{2}\left(\mathbb{R}, \mathbb{R}^{2 N}\right)$ with $\mathcal{D}(A) \subset H^{1}\left(\mathbb{R}, \mathbb{R}^{2 N}\right)$. In order to establish a variational setting for the system $(H S)$, we have the following Lemmas due to [21].

Lemma 2.1. ([21], Lemma 2.2). Suppose $\left(L_{0}\right)$ holds. Then $\sigma(A)=\sigma_{d}(A)$.

From Lemma 2.1, we know that the Hamiltonian operator $A$ has a sequence of eigenvalues

$$
\cdots \lambda_{-k} \leq \cdots \leq \lambda_{-1} \leq 0<\lambda_{1} \leq \cdots \leq \lambda_{k} \cdots
$$

with $\lambda_{ \pm k} \rightarrow \pm \infty$ as $k \rightarrow \infty$, and corresponding eigenfunctions $\left\{e_{ \pm k}\right\}_{k \in \mathbb{N}}$ form an orthogonal basis in $L^{2}$. Observe that we have an orthogonal decomposition

$$
L^{2}=L^{-} \oplus L^{0} \oplus L^{+}, \text {and } z=z^{-}+z^{0}+z^{+},
$$

such that $A$ is negative definite on $L^{-}$and positive definite on $L^{+}$and $L^{0}=\operatorname{ker} A$. Let $P^{0}: L^{2} \rightarrow L^{0}$ be the projection. Set $E:=\mathcal{D}\left(|A|^{\frac{1}{2}}\right)$ be the domain of the selfadjoint 
operator $|A|^{\frac{1}{2}}$ which is a Hilbert space equipped with the inner product

$$
\langle z, w\rangle=\left(|A|^{\frac{1}{2}} z,|A|^{\frac{1}{2}} w\right)_{2}+\left(P^{0} z, P^{0} w\right)_{2}
$$

and norm $\|z\|=\langle z, z\rangle^{\frac{1}{2}}$. Let $E^{ \pm}:={\overline{\operatorname{span}\left\{e_{ \pm k}\right\}_{k \in \mathbb{N}}}}, E^{0}=\operatorname{ker} A$. Clearly, $E^{-}, E^{0}$ and $E^{+}$are orthogonal with respect to the products $(\cdot, \cdot)_{2}$ and $\langle\cdot, \cdot\rangle$. Hence

$$
E=E^{-} \oplus E^{0} \oplus E^{+}
$$

is an orthogonal decomposition of $E$. Moreover, it is easy to prove the following embedding theorem by Lemma 2.1 .

Lemma 2.2. ([21], Lemma 2.3). E embeds continuously into $H^{\frac{1}{2}}:=H^{\frac{1}{2}}\left(\mathbb{R}, \mathbb{R}^{2 N}\right)$. Moreover, $E$ embeds compactly into $L^{p}:=L^{p}\left(\mathbb{R}, \mathbb{R}^{2 N}\right)$ for all $p \in[2, \infty)$, where $H^{\frac{1}{2}}$ is fractional order Sobolev spaces.

Next, On $E$ we define the following functional

$$
\Phi(z)=\frac{1}{2}\left(\left\|z^{+}\right\|^{2}-\left\|z^{-}\right\|^{2}\right)-\Psi(z),
$$

where $\Psi(z)=\int_{\mathbb{R}} R(t, z)$. Lemma 2.1 implies that $\Phi$ is strongly indefinite, and our hypotheses imply that $\Phi \in C^{1}(E, \mathbb{R})$, and a standard argument shows that critical points of $\Phi$ are solutions of system $(H S)$ (see [12]).

Now, we introduce the generalized Nehari manifold method. From above argument, we know $E$ is a Hilbert space with norm $\|\cdot\|$, and have an orthogonal decomposition

$$
E=E^{-} \oplus E^{0} \oplus E^{+}
$$

and $\operatorname{dim} E^{ \pm}=\infty, \operatorname{dim} E^{0}<\infty$. We denote by $S^{+}$the unit sphere in $E^{+}$, that is

$$
S^{+}:=\left\{z \in E^{+}:\|z\|=1\right\} .
$$

For $z=z^{+}+z^{0}+z^{-} \in E$, where $z^{ \pm} \in E^{ \pm}, z^{0} \in E^{0}$, we define

$$
E(z):=\mathbb{R} z \oplus\left(E^{-} \oplus E^{0}\right) \equiv \mathbb{R} z^{+} \oplus\left(E^{-} \oplus E^{0}\right),
$$

and

$$
\hat{E}(z):=\mathbb{R}^{+} z \oplus\left(E^{-} \oplus E^{0}\right) \equiv \mathbb{R}^{+} z^{+} \oplus\left(E^{-} \oplus E^{0}\right) .
$$

We make the following assumptions on $\Phi$ defined in (2.1):

$\left(A_{1}\right) \Psi(0)=0, \frac{1}{2}\left\langle\Psi^{\prime}(z), z\right\rangle>\Psi(z)>0$ for all $z \neq 0$ and $\Psi$ is weakly lower semicontinuous. 
$\left(A_{2}\right)$ For each $z \in E \backslash\left(E^{0} \oplus E^{-}\right)$, there exists a unique nontrivial critical point of $\hat{m}(z)$ of $\left.\Phi\right|_{\hat{E}(z)}$. Moreover, $\hat{m}(z)$ is the unique global maximum of $\left.\Phi\right|_{\hat{E}(z)}$.

$\left(A_{3}\right)$ There exists $\delta>0$ such that $\left\|\hat{m}(z)^{+}\right\| \geq \delta$ for all $z \in E \backslash\left(E^{0} \oplus E^{-}\right)$, and for each compact subset $\mathcal{K} \subset E \backslash\left(E^{0} \oplus E^{-}\right)$there exists a constant $C_{\mathcal{K}}$ such that $\|\hat{m}(z)\| \leq C_{\mathcal{K}}$.

We consider the following set introduced by Pankov [26]:

$$
\mathcal{M}:=\left\{z \in E \backslash\left(E^{0} \oplus E^{-}\right):\left\langle\Phi^{\prime}(z), z\right\rangle=0 \text { and }\left\langle\Phi^{\prime}(z), v\right\rangle=0 \forall v \in E^{0} \oplus E^{-}\right\} .
$$

Following Szulkin and Weth [27], we will call the set $\mathcal{M}$ the generalized Nehari manifold. Note that, if $z \neq 0$ and $\Phi^{\prime}(z)=0$, then $\Phi(z)=\Phi(z)-\frac{1}{2}\left\langle\Phi^{\prime}(z), z\right\rangle=$ $\frac{1}{2}\left\langle\Psi^{\prime}(z), z\right\rangle-\Psi(z)>0$ while $\Phi(\cdot)<0$ on $E^{0} \oplus E^{-}$. Hence $\mathcal{M}$ contains all nontrivial critical points of $\Phi$, and $\hat{E}(z) \cap \mathcal{M}=\{\hat{m}(z)\}$ whenever $z \in E \backslash\left(E^{0} \oplus E^{-}\right)$by $\left(A_{2}\right)$.

To prove our result, we define the mappings:

$$
\hat{m}: E \backslash\left(E^{0} \oplus E^{-}\right) \rightarrow \mathcal{M}, z \mapsto \hat{m}(z) \text { and } m:=\left.\hat{m}\right|_{S^{+}} .
$$

The following three results are due to Proposition 4.1, 4.2 and Corollary 4.3 of Szulkin and Weth. The proofs are given in Szulkin and Weth [27], here we omit the details.

Lemma 2.3. Assume that $\left(A_{1}\right),\left(A_{2}\right)$ and $\left(A_{3}\right)$ are satisfied. Then

(a) $\hat{m}$ is continuous.

(b) $m$ is a homeomorphism between $S^{+}$and $\mathcal{M}$.

Let

$$
\hat{I}: E^{+} \backslash\{0\} \rightarrow \mathbb{R}, \hat{I}(z):=\Phi(\hat{m}(z)) \text { and } I:=\left.\hat{I}\right|_{S^{+}} .
$$

Lemma 2.4. Assume that $\left(A_{1}\right),\left(A_{2}\right)$ and $\left(A_{3}\right)$ are satisfied. Then $\hat{I}$ is of class $C^{1}$ and

$$
\left\langle\hat{I}^{\prime}(z), w\right\rangle:=\frac{\left\|\hat{m}(z)^{+}\right\|}{\|z\|}\left\langle\Phi^{\prime}(z), w\right\rangle, \text { for all } z, w \in E^{+}, z \neq 0
$$

Corollary 2.5. Assume that $\left(A_{1}\right),\left(A_{2}\right)$ and $\left(A_{3}\right)$ are satisfied. Then (a) $I \in C^{1}\left(S^{+}, \mathbb{R}\right)$ and

$$
\left\langle\hat{I}^{\prime}(z), w\right\rangle:=\left\|\hat{m}(z)^{+}\right\|\left\langle\Phi^{\prime}(z), w\right\rangle \text { for all } w \in T_{z}\left(S^{+}\right),
$$

where $T_{z}\left(S^{+}\right)$is the tangent space of $S^{+}$at $z$.

(b) If $\left\{z_{n}\right\}$ is a Palais-Smale sequence for $I$, then $\left\{m\left(z_{n}\right)\right\}$ is a Palais-Smale sequence for $\Phi$. If $\left\{z_{n}\right\} \subset \mathcal{M}$ is a bounded Palais-Smale sequence for $\Phi$, then $\left\{m^{-1}\left(z_{n}\right)\right\}$ is a Palais-Smale sequence for $I$.

(c) $z$ is a critical point of $I$ if and only if $m(z)$ is a nontrivial critical point of $\Phi$. Moreover, the corresponding critical values coincide and $\inf _{S^{+}} I=\inf _{\mathcal{M}} \Phi$. 


\section{The Proof of Theorem}

Before giving the proof of the main theorem, we need some preliminary results.

Lemma 3.1. Assume that $\left(R_{1}\right),\left(R_{2}\right)$ and $\left(R_{3}\right)$ are satisfied. Then $\left(A_{1}\right)$ is satisfied.

Proof. Observe that, by $\left(R_{1}\right)-\left(R_{3}\right)$ we have $\Psi(0)=0$ and

$$
\frac{1}{2}\left\langle\Psi^{\prime}(z), z\right\rangle>\Psi(z)>0, \forall z \neq 0 .
$$

Let $z_{n} \rightarrow z$ in $E$, Lemma 2.2 implies $z_{n} \rightarrow z$ in $L^{2}$, hence $z_{n} \rightarrow z$ a.e. on $\mathbb{R}$. By Fatou's lemma we obtain

$$
\begin{aligned}
\Psi(z) & =\int_{\mathbb{R}} R(t, z)=\int_{\mathbb{R}} \lim _{n \rightarrow \infty} R\left(t, z_{n}\right) \\
& \leq \liminf _{n \rightarrow \infty} \int_{\mathbb{R}} R\left(t, z_{n}\right)=\liminf _{n \rightarrow \infty} \Psi\left(z_{n}\right) .
\end{aligned}
$$

This proves that $\Psi$ is weakly lower semicontinuous.

Lemma 3.2. Let $\left(L_{0}\right),\left(R_{1}\right),\left(R_{2}\right)$ and $\left(R_{3}\right)$ be satisfied. Then there exists $R>0$ such that $\Phi(\cdot) \leq 0$ on $E(z) \backslash B_{R}(0)$ for every $z \in E^{+} \backslash\{0\}$.

Proof. We modify the proof of [25] since $E^{0} \neq\{0\}$. For the completeness, we give the details here. Since $E(z)=E(z /\|z\|)$, we may assume that $\|z\|=1$ for every $z \in E^{+} \backslash\{0\}$. Suppose to the contradiction that there exists a sequence $z_{n} \in E^{+} \backslash\{0\}$ and $w_{n} \in E\left(z_{n}\right)$ such that $\Phi\left(w_{n}\right)>0$ for all $n$ and $\left\|w_{n}\right\| \rightarrow \infty$ as $n \rightarrow \infty$. Passing to a subsequence, we may assume that $z_{n} \rightarrow z \in E^{+},\|z\|=1$. Set $v_{n}=\frac{w_{n}}{\left\|w_{n}\right\|}=s_{n} z_{n}+v_{n}^{-}+v_{n}^{0}$, then $1=\left\|v_{n}\right\|^{2}=s_{n}^{2}+\left\|v_{n}^{-}\right\|^{2}+\left\|v_{n}^{0}\right\|^{2}$ and

$$
0<\frac{\Phi\left(w_{n}\right)}{\left\|w_{n}\right\|^{2}}=\frac{1}{2}\left(s_{n}^{2}-\left\|v_{n}^{-}\right\|^{2}\right)-\int_{\mathbb{R}} \frac{R\left(t, w_{n}\right)}{\left|w_{n}\right|^{2}}\left|v_{n}\right|^{2} .
$$

By $\left(R_{1}\right)$, we know $R(t, z) \geq 0$ and have

$$
\left\|v_{n}^{-}\right\|^{2} \leq s_{n}^{2}=1-\left\|v_{n}^{-}\right\|^{2}-\left\|v_{n}^{0}\right\|^{2} .
$$

If $s_{n} \rightarrow s>0$, going to a subsequence if necessary, we may assume $v_{n} \rightarrow v$ and $v_{n}(x) \rightarrow v(x)$ a.e. on $\mathbb{R}$. Hence $v=s z+v^{-}+v^{0} \neq 0$, therefore $\left|w_{n}\right|=|| w_{n}||\left|v_{n}\right| \rightarrow$ $\infty$. By $\left(R_{1}\right),\left(R_{3}\right)$ and Fatou's lemma, we have

$$
\begin{aligned}
0 & \leq \lim _{n \rightarrow \infty} \frac{\Phi\left(w_{n}\right)}{\left\|w_{n}\right\|^{2}}=\lim _{n \rightarrow \infty}\left(\frac{1}{2}\left(s_{n}^{2}-\left\|v_{n}^{-}\right\|^{2}\right)-\int_{\mathbb{R}} \frac{R\left(t, w_{n}\right)}{\left|w_{n}\right|^{2}}\left|v_{n}\right|^{2}\right) \\
& \leq \frac{1}{2}-\liminf _{n \rightarrow \infty} \int_{\mathbb{R}} \frac{R\left(t, w_{n}\right)}{\left|w_{n}\right|^{2}}\left|v_{n}\right|^{2} \\
& \leq \frac{1}{2}-\int_{\mathbb{R}} \liminf _{n \rightarrow \infty} \frac{R\left(t, w_{n}\right)}{\left|w_{n}\right|^{2}}\left|v_{n}\right|^{2} \\
& =-\infty,
\end{aligned}
$$


which implies a contradiction. If $s_{n} \rightarrow 0$, so up to a subsequence, $v_{n}^{-} \rightarrow 0$ and $v_{n}^{0} \rightarrow v^{0} \neq 0$. hence $v=v^{0} \neq 0$. Then (3.2) follows again, which implies a contradiction.

Lemma 3.3. Suppose that $\left(R_{1}\right)-\left(R_{6}\right)$ be satisfied. Let $s \in \mathbb{R}, s \geq-1$ and $z, u \in \mathbb{R}^{2 N}$ with $w:=s z+u \neq 0$ and let $t \in \mathbb{R}$. Then

$$
R_{z}(t, z) \cdot\left(s\left(\frac{s}{2}+1\right) z+(s+1) u\right)+R(t, z)-R(t, w+z)<0 .
$$

Proof. We modify the proof of [25] and give the details for the completeness (see also [24]). We fix $t \in \mathbb{R}$ and $z, u \in \mathbb{R}^{2 N}$. Let $s \geq-1$ and $y=w+z=(s+1) z+u$,

$$
f(s):=R_{z}(t, z) \cdot\left(s\left(\frac{s}{2}+1\right) z+(s+1) u\right)+R(t, z)-R(t, y) .
$$

We need to show $f(s)<0$ whenever $w=s z+u \neq 0$. We first consider the case $z=0$, then $y \neq 0$ by $w \neq 0$, hence $f(s)=-R(t, y)<0$ by $\left(R_{1}\right)$. We may therefore assume $z \neq 0$ from now on. If $z \cdot y \leq 0$, from $\left(R_{3}\right)$ and $\left(R_{4}\right)$, we have

$$
\begin{aligned}
f(s) & =R_{z}(t, z) \cdot\left(s\left(\frac{s}{2}+1\right) z+(s+1)(y-(s+1) z)\right)+R(t, z)-R(t, y) \\
& =-\left(\frac{s^{2}}{2}+s+1\right) R_{z}(t, z) \cdot z+(s+1) R_{z}(t, z) \cdot y+R(t, z)-R(t, y) \\
& \leq-\frac{1}{2}(s+1)^{2} R_{z}(t, z) \cdot z+(s+1) R_{z}(t, z) \cdot y-R(t, y)<0 .
\end{aligned}
$$

Next we note that $\left(R_{1}\right)$ implies

$$
f(-1)=-\frac{1}{2} R_{z}(t, z) \cdot z+R(t, z)-R(t, y)<-R(t, y) \leq 0 .
$$

By $\left(R_{3}\right)$, it is easy to see that

$$
\lim _{s \rightarrow \infty} f(s)=-\infty
$$

Moreover,

$$
f^{\prime}(s)=R_{z}(t, z) \cdot y-R_{z}(t, y) \cdot z .
$$

Suppose that $f(s)$ must attain its maximum on $[-1, \infty)$ at some point $s_{0}$ with $f\left(s_{0}\right) \geq$ 0 . Then $f^{\prime}\left(s_{0}\right)=0$, and $z \cdot y>0$ by (3.3). Thus, by (3.4) and $\left(R_{6}\right)$, we have $|z|=|y|$, which together with $w \neq 0,\left(R_{3}\right)$ and $\left(R_{5}\right)$ imply

$$
\begin{aligned}
f\left(s_{0}\right) & =R_{z}(t, z) \cdot\left(s_{0}\left(\frac{s_{0}}{2}+1\right) z+\left(s_{0}+1\right)\left(y-\left(s_{0}+1\right) z\right)\right)+R(t, z)-R(t, y) \\
& =\left(s_{0}+1\right) R_{z}(t, z) \cdot y-\left(\frac{s_{0}^{2}}{2}+s_{0}+1\right) R_{z}(t, z) \cdot z \\
& <\left(s_{0}+1-\frac{s_{0}^{2}}{2}-s_{0}-1\right) R_{z}(t, z) \cdot z \\
& =-\frac{1}{2} s_{0}^{2} R_{z}(t, z) \cdot z \leq 0,
\end{aligned}
$$


which contradicts with $f\left(s_{0}\right) \geq 0$. Therefore, $f\left(s_{0}\right)<0$ whenever $w=s z+u \neq 0$.

Lemma 3.4. Suppose that $\left(R_{1}\right)-\left(R_{6}\right)$ be satisfied. Then

(i) for $z \in \mathcal{M}$, we have $\Phi(z+w)<\Phi(z)$, where $w \neq 0, w=s z+u, u \in E^{0} \oplus E^{-}$ and $s \geq-1$, and $z$ is the unique global maximum of $\left.\Phi\right|_{\hat{E}(z)}$;

(ii) for each $z \in E \backslash\left(E^{0} \oplus E^{-}\right)$, the set $\hat{E}(z) \cap \mathcal{M}$ consists of precisely one point $\hat{m}(z)$ which is unique global maximun of $\left.\Phi\right|_{\hat{E}(z)}$.

Proof. (i) For $z \in \mathcal{M}$, let $z+w \in \hat{E}(z)$ with $w \neq 0$. Then $z+w=(1+s) z+u$, where $s \geq-1$ and $u=u^{0}+u^{-} \in E^{0} \oplus E^{-}$. By (2.1) we have

$$
\begin{aligned}
& \Phi(z+w)-\Phi(z) \\
= & \frac{1}{2}\left(\left((1+s)^{2}-1\right)\langle A z, z\rangle+2(1+s)\langle A z, u\rangle+\langle A u, u\rangle\right)+\int_{\mathbb{R}} R(t, z)-R(t, z+w) \\
= & -\frac{\left\|u^{-}\right\|^{2}}{2}+\left\langle A z, s\left(\frac{s}{2}+1\right) z+(1+s) u\right\rangle+\int_{\mathbb{R}} R(t, z)-R(t, z+w) \\
= & -\frac{\left\|u^{-}\right\|^{2}}{2}+\int_{\mathbb{R}}\left[R_{z}(t, z) \cdot\left(s\left(\frac{s}{2}+1\right) z+(s+1) u\right)\right]+R(t, z)-R(t, z+w) .
\end{aligned}
$$

In the last step we have used the fact that $z \in \mathcal{M}$ and $\phi:=s\left(\frac{s}{2}+1\right) z+(s+1) u \in E(z)$, therefore

$$
0=\left\langle\Phi^{\prime}(z), \phi\right\rangle=\langle A z, \phi\rangle-\int_{\mathbb{R}} R_{z}(t, z) \cdot \phi .
$$

Since $w$ is nonzero on a set of positive measure, the last integral above is negative according to Lemma 3.3 and hence $\Phi(z+w)<\Phi(z)$.

(ii) Similar to the proof of Lemma 2.6 of Szulkin and Weth [25]. It is easy to prove the above conclusion (ii) by conclusion (i) and Lemma 3.2. Here we omit the details of proof.

From the preceding Lemma, we have the following Lemma.

Lemma 3.5. Suppose that $\left(R_{1}\right)-\left(R_{6}\right)$ be satisfied. Then $\left(A_{2}\right)$ is satisfied.

Lemma 3.6. Suppose that $\left(R_{1}\right)-\left(R_{6}\right)$ be satisfied. Then $\left(A_{3}\right)$ is satisfied.

Proof. Obviously, $\left(R_{2}\right)$ implies $\Psi^{\prime}(z)=o(\|z\|)$ as $|z| \rightarrow 0$, which together with $\left(A_{1}\right)$ imply that for any $\varepsilon>0$ and $z \in E^{+}, \Psi(z) \leq \frac{\varepsilon}{2}\|z\|^{2}$ when $|z|<\delta$ for some $\delta>$ 0 . Hence we can find $\rho, \eta>0$ such that $\Phi(z) \geq \eta$ for any $z \in\left\{z \in E^{+}:\|z\|=\rho\right\}$. By $\left(A_{2}\right), \Phi(\hat{m}(z)) \geq \eta$ for any $z \in E \backslash\left(E^{0} \oplus E^{-}\right)$. Since $\Psi(\cdot) \geq 0$, by (2.1) we have $\eta \leq \Phi(\hat{m}(z))=\frac{1}{2}\left(\left\|\hat{m}(z)^{+}\right\|^{2}-\left\|\hat{m}(z)^{-}\right\|^{2}\right)-\Psi(\hat{m}(z)) \leq \frac{1}{2}\left(\left\|\hat{m}(z)^{+}\right\|^{2}-\left\|\hat{m}(z)^{-}\right\|^{2}\right)$.

Hence $\left\|\hat{m}(z)^{+}\right\| \geq \sqrt{2 \eta}$. 
Now let $\mathcal{K}$ be a compact subset of $E \backslash\left(E^{0} \oplus E^{-}\right)$. We want to show that there exists a constant $C_{\mathcal{K}}$ such that $\|\hat{m}(z)\| \leq C_{\mathcal{K}}, \forall z \in \mathcal{K}$. Since $\hat{m}(z)=\hat{m}\left(z^{+} /\left\|z^{+}\right\|\right)$ $\forall z \in \mathcal{K}$, we may assume that $\mathcal{K} \subset S^{+}$. Suppose by contradiction that there exists a sequence $\left\{z_{n}\right\} \subset \mathcal{K}$ such that $\left\|\hat{m}\left(z_{n}\right)\right\| \rightarrow \infty$. Since $\hat{m}\left(z_{n}\right) \in \hat{E}\left(z_{n}\right)$, let $v_{n}=$ $\frac{\hat{m}\left(z_{n}\right)}{\left\|\hat{m}\left(z_{n}\right)\right\|}=s_{n} z_{n}+v_{n}^{-}+v_{n}^{0}$, then $1=\left\|v_{n}\right\|^{2}=s_{n}^{2}+\left\|v_{n}^{-}\right\|^{2}+\left\|v_{n}^{0}\right\|^{2}$. Similar to (3.1) we have

$$
\left\|v_{n}^{-}\right\|^{2} \leq s_{n}^{2}=1-\left\|v_{n}^{-}\right\|^{2}-\left\|v_{n}^{0}\right\|^{2} .
$$

Hence, by the same fashion as the last part of the proof of Lemma 3.2, we get a contradiction.

Lemma 3.7. Under the assumptions of Theorem 1.1. Then $\Phi$ satisfies the PalaisSmale condition on $\mathcal{M}$.

Proof. Let $\left\{z_{n}\right\} \subset \mathcal{M}$ a sequence such that $\Phi\left(z_{n}\right) \leq c$ for some $c>0$ and $\Phi^{\prime}\left(z_{n}\right) \rightarrow 0$. If $\left\{z_{n}\right\}$ is unbounded, we set $w_{n}=\frac{z_{n}}{\left\|z_{n}\right\|}$. Passing to a subsequence, we may assume $\left\|z_{n}\right\| \rightarrow \infty$ and $w_{n} \rightarrow w$. If $w \neq 0$, it follows from (2.1) that

$$
0 \leq \frac{\Phi\left(z_{n}\right)}{\left\|z_{n}\right\|^{2}}=\frac{1}{2}\left(\left\|w_{n}^{+}\right\|^{2}-\left\|w_{n}^{-}\right\|^{2}\right)-\int_{\mathbb{R}} \frac{R\left(t, z_{n}\right)}{\left|z_{n}\right|^{2}}\left|w_{n}\right|^{2} .
$$

By $w \neq 0$, then $\left|z_{n}\right| \rightarrow \infty$. By $\left(R_{1}\right),\left(R_{3}\right)$ and Fatou's lemma we have

$$
\int_{\mathbb{R}} \frac{R\left(t, z_{n}\right)}{\left|z_{n}\right|^{2}}\left|w_{n}\right|^{2} \rightarrow \infty
$$

which implies $0 \leq-\infty$. Hence $w=0$. By (3.5) and $\left(R_{1}\right)$, we have $\left\|w_{n}^{+}\right\| \geq\left\|w_{n}^{-}\right\|$. If $w_{n}^{+} \rightarrow 0$, then also $w_{n}^{-} \rightarrow 0$ and therefore

$$
\left\|w_{n}^{0}\right\|^{2}=1-\left\|w_{n}^{+}\right\|^{2}-\left\|w_{n}^{-}\right\|^{2} \rightarrow 1 .
$$

Hence $w_{n}^{0} \rightarrow w^{0}$ because $\operatorname{dim} E^{0}<\infty$. So $w \neq 0$, a contradiction. Therefore $w_{n}^{+} \nrightarrow 0$ and $\left\|w_{n}^{+}\right\| \geq \alpha$ for all $n$ and some $\alpha>0$. It is clear that $s w_{n}^{+} \in \hat{E}\left(z_{n}\right)$ for all $s>0$. By Lemma 3.4 or $\left(A_{2}\right)$, we have

$$
c \geq \Phi\left(z_{n}\right) \geq \Phi\left(s w_{n}^{+}\right) \geq \frac{1}{2} s^{2} \alpha^{2}-\Psi\left(s w_{n}^{+}\right) .
$$

Since $w_{n}^{+} \rightarrow 0$, we deduce from the Lemma 2.2 that $w_{n}^{+} \rightarrow 0$ in $L^{p}$ for $p \geq 2$. Therefore, by $\left(R_{2}\right)$ we deduce that $\Psi\left(s w_{n}^{+}\right) \rightarrow 0$. It then follows that $c \geq \frac{1}{2} s^{2} \alpha^{2}$ for all $s>0$. This gives another contradiction if we take $s$ large enough. Hence $\left\{z_{n}\right\}$ is bounded.

By taking a subsequence if necessary we have $z_{n} \rightarrow z$ in $\mathcal{M}$. It follows from the Lemma 2.2 that $z_{n} \rightarrow z$ in $L^{p}$ for $p \geq 2$. Now we easily obtain from $\left(R_{2}\right)$ 


$$
\begin{aligned}
& \int_{\mathbb{R}}\left(R_{z}\left(t, z_{n}\right)-R_{z}(t, z)\right)\left(z^{+}-z_{n}^{+}\right) \\
\leq & \int_{\mathbb{R}}\left|R_{z}\left(t, z_{n}\right)-R_{z}(t, z)\right|\left|z^{+}-z_{n}^{+}\right| \\
\leq & \int_{\mathbb{R}}\left(\varepsilon\left(|z|+\left|z_{n}\right|\right)+C_{\varepsilon}\left(|z|^{p-1}+\left|z_{n}\right|^{p-1}\right)\right)\left|z^{+}-z_{n}^{+}\right|
\end{aligned}
$$

for any $\varepsilon>0$ and some $C_{\varepsilon}>0$. By Holder inequality we have

$$
\int_{\mathbb{R}}\left(\varepsilon\left(|z|+\left|z_{n}\right|\right)+C_{\varepsilon}\left(|z|^{p-1}+\left|z_{n}\right|^{p-1}\right)\right)\left|z^{+}-z_{n}^{+}\right| \rightarrow 0 .
$$

By (3.6) we have

$$
\begin{aligned}
o(1) & =\left\langle\Phi^{\prime}(z)-\Phi^{\prime}\left(z_{n}\right), z^{+}-z_{n}^{+}\right\rangle \\
& =\left\|z^{+}-z_{n}^{+}\right\|^{2}+\int_{\mathbb{R}}\left(R_{z}\left(t, z_{n}\right)-R_{z}(t, z)\right)\left(z^{+}-z_{n}^{+}\right) \\
& =\left\|z^{+}-z_{n}^{+}\right\|^{2}+o(1),
\end{aligned}
$$

this implies $z_{n}^{+} \rightarrow z^{+}$as $n \rightarrow \infty$. Similarly, we can prove that $z_{n}^{-} \rightarrow z^{-}$as $n \rightarrow \infty$. Since $\operatorname{dim} E^{0}<\infty, z_{n}^{0} \rightarrow z^{0}$ as $n \rightarrow \infty$. So $z_{n} \rightarrow z$ as $n \rightarrow \infty$ in $\mathcal{M}$.

We also need the following consequence of the Ekeland variational principle due to [31]:

Lemma 3.8. Let $E$ be a Banach space and let $\varphi \in C^{1}(E, \mathbb{R})$ be bounded below. If $\varphi$ satisfies the Palais-Smale condition at level $c:=\inf _{E} \varphi$, then there exists $u \in E$ such that $\varphi^{\prime}(u)=0$ and $c=\varphi(u)$.

Proof of Theorem 1.1. We already know from Lemmas 3.1, 3.5 and 3.6 that $\left(A_{1}\right)-\left(A_{3}\right)$ are satisfied. By Corollary 2.5 -(a) $I \in C^{1}\left(S^{+}, \mathbb{R}\right)$.

First, we show that $I$ satisfies the Palais-Smale condition on $S^{+}$. Let $\left\{z_{n}\right\}$ be a Palais-Smale sequence for $I$. By Corollary 2.5-(b) $\left\{m\left(z_{n}\right)\right\}$ is a Palais-Smale sequence for $\Phi$ on $\mathcal{M}$. By Lemma 3.7 we have $m\left(z_{n}\right) \rightarrow z$ up to a subsequence. Since $m^{-1}$ is continuous, it follows that $z_{n} \rightarrow m^{-1}(z)$. Hence $I$ satisfies the palais-Smale condition on $S^{+}$. Particularly, $I$ satisfies the Palais-Smale condition at level $c=\inf _{S^{+}} I$. By Corollary 2.5-(c) $\inf _{S^{+}} I=\inf _{\mathcal{M}} \Phi>0$ and $I$ is bounded below. By Lemma 3.8 $\inf _{S^{+}} I$ is a critical value of $I$. There then exists $z_{0} \in S^{+}$such that $I\left(z_{0}\right)=\inf _{S^{+}} I$ and $I^{\prime}\left(z_{0}\right)=0$. It follows from Corollary 2.5-(c) that $m\left(z_{0}\right)$ is a critical point of $\Phi$ and $\Phi\left(m\left(z_{0}\right)\right)=\inf _{\mathcal{M}} \Phi$. Hence $m\left(z_{0}\right)$ is a ground state solution of system $(H S)$. 


\section{REFERENCES}

1. V. Coti-Zelati, I. Ekeland and E. Séré, A variational approach to homoclinic orbits in Hamiltonian systems, Math. Ann., 228 (1990), 133-160.

2. V. Coti-Zelati and P. Rabinowitz, Homoclinic orbits for second order Hamiltonian systems possessing superqudratic potentials, J. Amer. Math. Soc., 4 (1991), 693-727.

3. E. Séré, Existence of infinitely many homoclinic orbits in Hamiltonian systems, Math. Z., 209 (1992), 27-42.

4. Y. H. Ding, Existence and multiciplicity results for homoclinic solutions to a class of Hamiltonian systems, Nonlin. Anal., 25 (1995), 1095-1113.

5. Y. H. Ding, Multiple homoclinics in a Hamiltonian system with asymptotically or super linear terms, Commun. Contemp. Math., 4 (2006), 453-480.

6. Y. H. Ding and M. Girardi, Periodic and homoclinic solutions to a class of Hamiltonian systems with the potentials changing sign, Dyn. Sys. Appl., 2 (1993), 131-145.

7. Y. H. Ding and M. Girardi, Infinitely many homoclinic orbits of a Hamiltonian system with symmetry, Nonlinear Anal., 38 (1999), 391-415.

8. Y. H. Ding and L. Jeanjean, Homoclinic orbits for nonperiodic Hamiltonian system, $J$. Differential Equations, 237 (2007), 473-490.

9. Y. H. Ding and S. J. Li, Homoclinic orbits for first order Hamiltonian systems, J. Math. Anal. Appl., 189 (1995), 585-601.

10. Y. H. Ding and C. Lee, Existence and exponential decay of homoclinics in a nonperiodic superquadrtic Hamiltonian system, J. Differential Equations, 246 (2009), 2829-2848.

11. Y. H. Ding and M. Willem, Homoclinic orbits of a Hamiltonian system, Z. Angew. Math. Phys., 50 (1999), 759-778.

12. Y. H. Ding, Variational Methods for Strongly Indefinite Problems, World Scientific Press, 2008.

13. H. Hofer and K. Wysocki, First order ellipic systems and the existence of homoclinic orbits in Hamiltonian systems, Math. Ann., 228 (1990), 483-503.

14. W. Omana and M. Willem, Homoclinic orbits for a class of Hamiltonian systems, Diff. and Int., 5 (1992), 1115-1120.

15. G. W. Chen and S. W. Ma, Homoclinic orbits of superquadratic Hamiltonian system, Proc. Amer. Math. Soc., 139 (2011), 3973-3983.

16. P. H. Rabinowitz, Homoclinic orbits for a class of Hamiltonian systems, Proc. Roy. Soc. Edinburgh, 114 (1990), 33-38.

17. P. H. Rabinowitz and K. Tanaka, Some results on connecting orbits for a class of Hamiltonian systems, Math. Z., 206 (1991), 473-499.

18. A. Szulkin and W. Zou, Homoclinic orbits for asymptotically linear Hamiltonian systems, J. Funct. Anal., 187 (2001), 25-41. 
19. K. Tanaka, Homoclinic orbits in a first order superquadratic Hamiltonian system: Convergence of subharmonic orbits, J. Diff. Eqs., 94 (1991), 315-339.

20. J. Wang, J. X. Xu and F. B. Zhang, Homoclinic orbits for superlinear Hamiltonian systems without Ambrostti-Rabinowitz growth condition, Disc. Conti. Dyna. Syst. Ser. A, 27 (2010), 1241-1257.

21. W. P. Qin, J. Zhang and F. K. Zhao, Homoclinic orbits for a class of nonperiodic Hamiltonian systems, Abst. Appl. Anal., Vol. 2012, ID 769232.

22. F. K. Zhao, L. G. Zhao and Y. H. Ding, Multiple solution for a superlinear and periodic ellipic system on $\mathbb{R}^{N}, Z$. Angew. Math. Phys., 62 (2011), 495-511.

23. S. B. Liu, On superlinear Schrodinger equations with periodic potential, Calc. Var. Part. Diffe. Equ., 45 (2012), 1-9.

24. G. W. Chen and S. W. Ma, Periodic solutions for Hamiltonian systems without AmbrosettiRabinowitz condition and spectrum 0, J. Math. Anal. Appl., 379 (2011), 842-851.

25. A. Szulkin and T. Weth, Ground state solutions for some indefinite problems, J. Funct. Anal., 257 (2009), 3802-3822.

26. A. Pankov, Periodic nonlinear Schrödinger equation with application to photonic crystals, Milan J. Math., 73 (2005), 259-287.

27. A. Szulkin and T. Weth, The method of Nehari manifold. Handbook of nonconvex analysis and applications, 597632, Int. Press, Somerville, 2010.

28. M. B. Yang, W. X. Chen and Y. H. Ding, Solutions for periodic Schrodinger equation with spectrum zero and general superlinear nonlinearities, J. Math. Anal. Appl., 364 (2010), 404-413.

29. M. B. Yang, Ground state solutions for a periodic Schroinger equationwith superlinear nonlinearities, Nonlinear Anal., 72 (2010), 2620-2627.

30. M. Schechter and W. M. Zou, Weak linking theorems and Schrodinger equations with critical Sobolev exponent, ESAIM Control Optim. Calc. Var., 9 (2003), 601-619.

31. M. Willem, Minimax Theorems, Birkhäuser, Berlin, 1996.

Jian Zhang, Xianhua Tang and Wen Zhang

School of Mathematics and Statistics

Central South University

Changsha 410083, Hunan

P. R. China

E-mail: zhangjian433130@163.com

tangxh@mail.csu.edu.cn

zwmath2011@163.com 